\title{
An intensive multiagent chemotherapy regimen for brain tumours occurring in very young children
}

Linda S Lashford, Richard H A Campbell, H Rao Gattamaneni, Kath Robinson, David Walker, Cliff Bailey

\begin{abstract}
Standard treatment for the majority of malignant brain tumours consists of surgery and radiotherapy. This treatment has late morbidity which is accentuated in the very young child. As part of a strategy to improve quality of life and overall survival of young children with brain tumours, members of the United Kingdom Children's Cancer Study Group (UKCCSG) have piloted an intensive chemotherapy regimen which aims to avoid or delay radiotherapy following surgery. Twenty eight children with a variety of malignant brain tumours have received the regimen, which contains carboplatin, vincristine, cyclophosphamide, methotrexate, and cisplatin. The treatment is toxic, resulting in one death from infection. The bulk of the toxicity was associated with the administration of carboplatin. All but three children eventually required adjuvant radiotherapy and this was given between 1.5 and 27 months from diagnosis (median delay to radiotherapy, 12 months). Using this treatment regimen, overall survival at four years is $35 \%$ (confidence intervals $10 \%$ to $60 \%$ ). While there is no evidence from this study that radiotherapy can be abandoned in the management of malignant brain tumours, its introduction may be delayed using suitable chemotherapy, thus allowing time for further CNS development. This treatment strategy has been taken forward as an international clinical trial run through the International Society for Paediatric Oncology, but using a smaller dose of carboplatin to reduce toxicity.

(Arch Dis Child 1996; 74: 219-223)
\end{abstract}

Keywords: brain tumours, chemotherapy.

For The United

Kingdom Children's

Cancer Study Group

(UKCCSG) Brain

Tumour Group

L S Lashford

R H A Campbell

H R Gattamaneni

K Robinson

D Walker

C Bailey

Correspondence to Dr Linda $S$ Lashford Children's Unit, Christie Hospital NHS Trust,

Wilmslow Road, Withington, Manchester M20 4BX.

Accepted 16 November 1995 and to resect tumour, followed by radiotherapy to treat residual tumour and control distant metastases. During the last 20 years the changes that have contributed to an improved mainly been advances in central nervous system imaging, neuroanaesthesia, perioperative care, and delivery of radiotherapy. ${ }^{1}$ The overall survival for children with brain tumours currently stands at $50 \%$, with the quality of that survival affected by a variety of neuropsychological, endocrine and skeletal late effects.

These issues are most sharply focused when caring for the very young child. For a number of the malignant brain tumours, young age is often an adverse prognostic factor. This is best shown by figures from population based cancer registries such as the British National Registry of Childhood Tumours. In the period 1971 to 1985, 548 children with spinal and brain tumours under the age of 2 were registered, accounting for $12 \%$ of all such tumours in childhood. ${ }^{2}$ Five year survival rates were poor at $20 \%$ for ependymoma and $13 \%$ for medulloblastoma. This compares with five year survival rates for all patients of $64 \%$ and $53 \%$ respectively. ${ }^{3} 4$ However, reports from various single centres suggest that similar cure rates to those obtained in older children can be achieved in young children provided that the same treatment is given. ${ }^{156}$ One explanation for the poorer survival in young children may be a reluctance to treat patients with full dose radiotherapy. It is well recognised that the major cause of collateral brain injury during treatment for childhood brain tumours is central nervous system (CNS) irradiation. ${ }^{78}$ The severity of subsequent neuropsychological deficit is directly correlated with CNS dose and younger age at treatment.

One way forward is to consider the role of adjuvant chemotherapy, either to delay or to avoid radiotherapy. This approach may improve overall survival while minimising treatment related brain injury. There is increasing evidence that many types of brain tumour are chemosensitive including medulloblastoma and other primitive neuroectodermal tumours (PNET), astrocytomas, and ependymomas. ${ }^{9-11}$ In an attempt to improve the prognosis of young children with brain tumours, members of the UKCCSG piloted an intensive chemotherapy regimen to be given after surgery. The purpose of the study was to assess the feasibility of giving the intensive regimen in very young children and to determine whether it was possible to delay radiotherapy. outcome in paediatric brain tumours have
Methods

Any child under the age of 3 years with a histologically proven malignant brain tumour not previously treated with radiotherapy and 
Chemotherapy schedule: cycle repeated up to $\times 7$

\begin{tabular}{|c|c|c|c|c|}
\hline & $\begin{array}{l}\text { Day } \\
0\end{array}$ & $\begin{array}{l}\text { Day } \\
14\end{array}$ & $\begin{array}{l}\text { Day } \\
28\end{array}$ & $\begin{array}{l}\text { Day } \\
42\end{array}$ \\
\hline $\begin{array}{l}\text { Vincristine }\left(1.5 \mathrm{mg} / \mathrm{m}^{2}\right) \\
\text { Carboplatin }\left(550 \mathrm{mg} / \mathrm{m}^{2} \times 2\right) \\
\text { Methotrexate }\left(8 \mathrm{~g} / \mathrm{m}^{2}\right) \\
\text { Cyclophosphamide }\left(1500 \mathrm{mg} / \mathrm{m}^{2}\right) \\
\text { Cisplatin }\left(40 \mathrm{mg} / \mathrm{m}^{2} \times 2\right)\end{array}$ & $\begin{array}{l}\star \\
\star \star\end{array}$ & $\begin{array}{l}\star \\
\star\end{array}$ & $\star$ & $\star \star$ \\
\hline
\end{tabular}

chemotherapy was eligible for the study. Following surgery, patients were staged to define the extent of the residual tumour. This comprised either computed tomographic (CT) scanning of the head and myelography, or nuclear magnetic resonance (NMR) imaging of the head and spine.

The chemotherapy schedule was designed to incorporate agents of proven activity against a range of CNS tumour types. The drugs were scheduled so that myelosuppressive agents were alternated with less myelosuppressive agents, leading to the design of repeated pulses of chemotherapy at 14 day intervals (table). This schedule results in the least myelosuppressive chemotherapy at a time of neutropenia and thrombocytopenia. Treatment was to be given on time unless life threatening complications supervened. Centres were encouraged to treat children on the chemotherapy schedule for a period of one year unless relapse or progression of the tumour occurred. Radiotherapy was recommended either at the time of relapse or in the case of biopsy proven residual disease at the end of the chemotherapy schedule. Local irradiation comprised a total dose of $50 \mathrm{~Gy}$. Craniospinal radiation was $25 \mathrm{~Gy}$ to children under 3 years and $35 \mathrm{~Gy}$ to children over the age of 3 , given for tumours that are known to metastasise within the neuroaxis.

All UKCCSG centres were surveyed and patients treated on the protocol were notified to one of the authors. In view of the intensity of the protocol, the first 62 cycles of chemotherapy were evaluated for the complications of treatment and duration of hospital stay. Centres were visited by one of the authors, the notes inspected, and toxicity graded by WHO criteria.

Children treated on the study were followed to time of radiotherapy, relapse or death

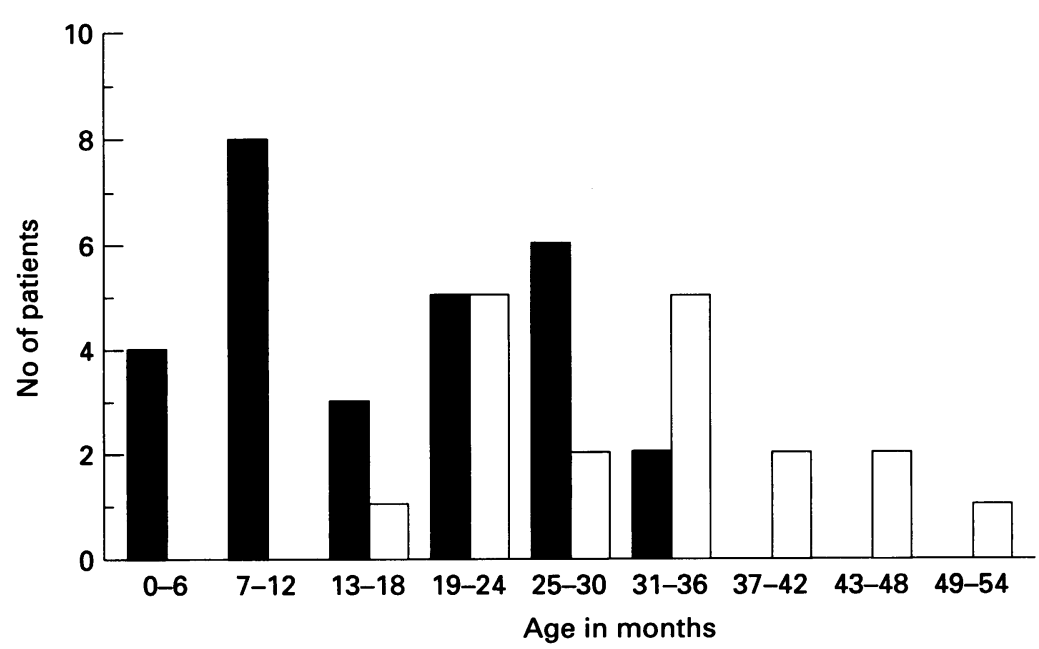

Figure 1 Black bars indicate the numbers of patients by age of presentation. Empty bars show the numbers of patients by age of irradiation. through the data monitoring service of the UKCCSG.

\section{Results}

Twenty eight children were treated on this study at a median age of 14.5 months (range: 1 to 33 months). The children presented the spectrum of malignant tumours seen in the very young child. These comprised 16 primitive neuroectodermal tumours (including medulloblastoma), five ependymomas, and seven others. In the latter group there were three children with choroid plexus carcinoma, three with malignant astrocytoma, and one meningeal sarcoma. There was an unusual distribution of primary sites in patients with a diagnosis of primitive neuroectodermal tumour (PNET), 39\% of tumours occurring at sites outside the posterior fossa. The majority of children $(75 \%)$ had an incomplete surgical resection (that is, residual abnormality on postoperative imaging suggestive of residual disease), three of whom received only biopsy. These were two patients with a PNET and one with glioblastoma. Imaging of the craniospinal axis revealed four patients with coexisting metastatic disease within the neuraxis.

Each full cycle of chemotherapy was projected to take 56 days, with 10 of these days spent in hospital for the chemotherapy to be given. The median time to administer cycles $1-6$ was $60,60,66,55,62$, and 64 days respectively. Most treatment cycles resulted in additional hospital admissions for complications of the chemotherapy. The median time spent in hospital for treatment related toxicity was 14 days and was largely attributable to infection (94 episodes); $37 \%$ of infective episodes were due to a febrile neutropenia in which no organism was identified, and in $21 \%$ an organism was cultured from serial blood samples. Three episodes were associated with severe sepsis, two with septic shock, and there was one infection related toxic death. Of particular note was the fact that the majority of infections were temporally related to the carboplatin induced neutropenia at the beginning of each cycle. Additionally, 14 treatment cycles were associated with significant haemorrhage, five with WHO grade 3-4 neurotoxicity, and 11 with WHO grade 3-4 pulmonary toxicity. Interestingly, eight episodes of pulmonary toxicity occurred in five patients with a pre-existing bulbar palsy. Of the 28 patients treated on the study, six died before radiotherapy could be given. These included the patient who died from sepsis and was shown to have persistent tumour at necropsy. Irradiation was not given to the remaining five patients either because of young age (one patient) or rapid progression of disease. Nineteen patients were eventually irradiated, six to the original site of the primary tumour and 13 with full craniospinal irradiation. The median time interval from initial surgery to radiotherapy was 12 months (range 1.5 to 27 months), resulting in a substantial shift in the distribution of age at irradiation (fig 1). This is further illustrated by the difference in the event-free and crude survival of this 


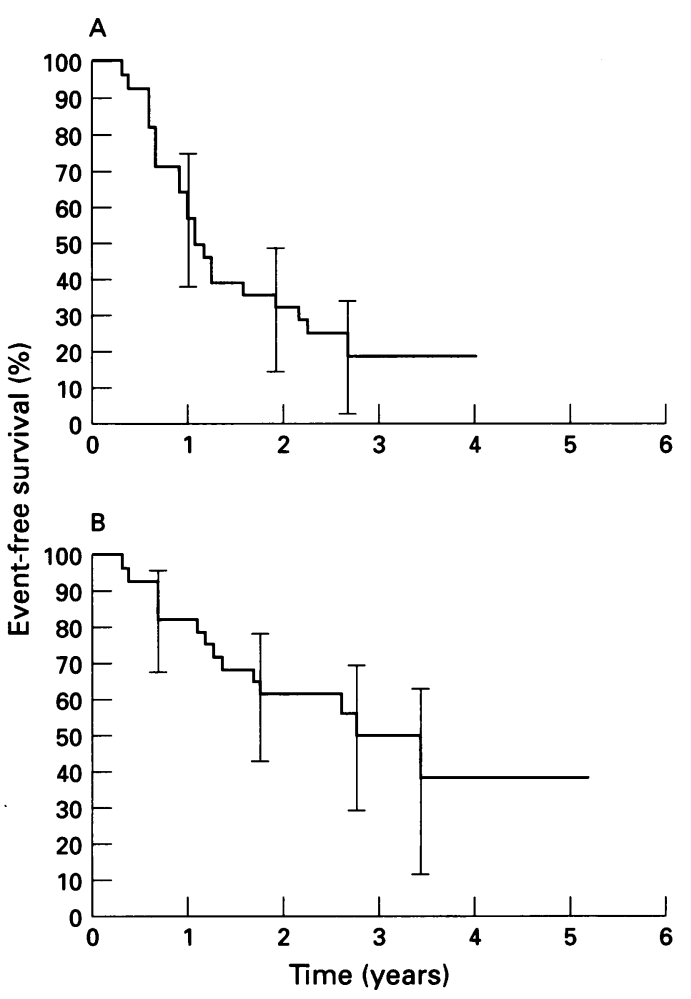

Figure 2 (A) Event-free survival for all patients in the study, with $95 \%$ confidence limits (Kaplan-Meier method). (B) Overall survival for all patients in the study, with $95 \%$ confidence intervals (Kaplan-Meier method).

group of patients (fig 2A,B). The most informative curve is the crude survival (2B), as the treatment strategy anticipates that a sizeable proportion of patients will relapse or progress, but may be rescued by delayed radiotherapy. Fourteen patients $(50 \%)$ are still alive at a median follow up of 35 months (range 26 to 62 ), of whom three are in continuous complete remission without ever being irradiated at 27, 31 , and 49 months from diagnosis (two with malignant astrocytoma and one PNET). Overall survival is $35 \%$ at 4 years, but has wide confidence intervals (10\% to $60 \%)$.

One group that warrants separate analysis comprises the patients with PNET. These patients require total CNS irradiation to control disease and are a group which would derive maximum benefit from an effective chemotherapy strategy. This group includes patients with medulloblastoma (10 patients) and six others with supratentorial primary tumours. Three of these patients were identified as having CNS metastatic disease at presentation. In 14 of these patients $(88 \%)$ the tumours either progressed or recurred, resulting in 12 patients being irradiated at between four and 23 months from diagnosis (median delay; 11 months). Eight patients remain alive, of whom one is alive at 31 months without ever having been irradiated.

\section{Discussion}

Central nervous system tumours account for between $20 \%$ and $25 \%$ of all childhood cancer and are thus the second most frequent malignancy of childhood. ${ }^{2}$ For the majority of children, irradiation of the primary tumour has improved local control of disease, while neuroaxis radiotherapy has prevented the emergence of leptomeningeal metastases in tumours such as medulloblastoma. ${ }^{12}$ The dose of irradiation, size of radiotherapy field, and age at radiotherapy are all important determinants in the development of collateral neuropsychological and soft tissue damage. The defects that occur are well described and include specific defects such as disorders of behaviour, dysphasia, dysgraphia, and spatial perception. ${ }^{13}$ The net result is a predicted loss in performance IQ of 25 points for patients treated with $35 \mathrm{~Gy}$ to the whole brain at 2 years of age. ${ }^{7}$ This is not an absolute figure but part of a continuum, with further loss occurring in younger individuals. Other developing tissues are also affected and include endocrine dysfunction secondary to hypothalamic, pituitary, and thyroid irradiation, as well as a reduction in spinal growth. ${ }^{14} 15$ These issues may influence decisions to treat patients with malignant CNS neoplasms in the first few years of life. In a British population based study spanning the years 1971-1985, $31 \%$ of children presenting under 2 years of age with a CNS tumour died either without treatment or within one month of surgery. ${ }^{2}$ Similar results have been reported from the USA, where young patients were found to die early and without effective treatment. ${ }^{16}$

In an attempt to improve the dismal prognosis for these children, various national groups are attempting to use chemotherapy either to delay or prevent CNS irradiation. One challenge is to define a chemotherapy schedule with activity against a broad range of tumours. The majority of treatment regimens include platinum analogues, cyclophosphamide, and vincristine, which have been shown to have activity against astrocytomas, PNETs, and ependymomas. ${ }^{17-20} \mathrm{~A}$ high dose infusion of methotrexate was included in this schedule to improve its CNS penetration ${ }^{21}$ and resulted in the design of a novel schedule which rapidly alternated myelosupressive drugs (carboplatin, cyclophosphamide) with less myelosuppressive drugs (methotrexate, cisplatin). The rationale of the schedule was to expose the tumour to multiple agents with different modes of action in an attempt to reduce the emergence of drug resistance. Such a regimen was recognised to be potentially toxic and so the main purpose of this study was to assess the feasibility of the approach. As such, no attempt was made to ascertain the number of children within the United Kingdom eligible for study but treated by other methods. The chemotherapy proved to be toxic, resulting in substantial need for hospital admission for infection and haemorrhage, as well as one toxic death. Much of the toxicity was related to the carboplatin, with more acute toxicity occurring during the first period of neutropenia and thrombocytopenia of each treatment cycle. Additional problems included the development of a severe respiratory illnesses in a small number of patients. Several drugs including methotrexate can cause or contribute to lung injury ${ }^{22}$ but this did not appear to be the case in this schedule, as it was clear that there was an excess of 
respiratory illness in patients with a bulbar palsy. It was felt that the recurrent respiratory distress was contributed to by repeated aspiration. Similarly, it was clear that neurotoxicity occurred because of shunt malfunction or progressive tumour, rather than drug related events. In general it was felt that the chemotherapy schedule was feasible, but because of the incidence of life threatening infection and haemorrhage following carboplatin it was recommended that future studies be undertaken with a reduced dose of this drug. These recommendations have been incorporated into an international multicentre study of the chemotherapy schedule for treatment of very young children with brain tumours administered by the International Society for Paediatric Oncology (SIOP) and the UKCCSG. The study opened in 1992 and is still recruiting patients.

The pilot patients have been monitored continuously to indicate any problems that are likely to emerge in the SIOP study. The small size of the pilot study makes it impossible to judge the efficacy of the treatment regimen. However, a few conclusions can be drawn which may aid in the development of future treatment approaches. Firstly, it is clear that it is possible to delay CNS radiotherapy for a substantial number of patients. The pilot study did not recommend routine irradiation at the end of chemotherapy unless there was histologically proven residual disease. This policy resulted in a median delay of 12 months from diagnosis to radiotherapy, allowing further CNS maturation to take place. At one extreme of outcome this has resulted in three patients who have not yet been irradiated, including one with a malignant astrocytoma in whom the tumour was only biopsied, and one with medulloblastoma who had residual disease at the end of treatment. However, the majority of patients eventually required irradiation to control CNS disease, suggesting that this chemotherapy schedule may be incapable of eradicating residual tumour. The majority of recent clinical protocols treating very young children with brain tumours take this view and incorporate guidelines for radiotherapy, at either a specified age or a specified duration of treatment. ${ }^{8}$ Less intensive chemotherapy is usually continued until radiotherapy is given. The current SIOP/UKCCSG study has continued with the philosophy developed in the pilot study and does not recommend routine irradiation at the end of chemotherapy in patients with no evidence of active tumour. This may be important, as the study enrols patients with a wide variety of primary brain tumours and in a larger cohort of patients it may be possible to identify a group who will benefit from chemotherapy alone. This issue is being closely monitored.

Various small single institution studies have suggested a role for preirradiation chemotherapy in the management of the young child. ${ }^{23} 24$ These studies have not only been valuable in indicating the validity of the treatment strategy but have shown that a wide range of paediatric brain tumours respond to chemotherapy. For example, the largest and perhaps most successful study of young children treated with pulsed chemotherapy and delayed irradiation has been run through the Paediatric Oncology Group (POG). This was a large multicentre study which enrolled 206 patients and produced an overall two year survival of $53 \% .{ }^{10}$ The study has also provided the best evidence to date that ependymomas are chemosensitive, recording a response rate of $48 \%$ to two cycles of vincristine and cyclophosphamide. Our pilot study indicates a similar two year survival of $58 \%$, but with wide confidence intervals and with evidence of continuing relapse. It will be necessary to await the outcome of the larger SIOP/UKCCSG study to define the efficacy of this treatment regimen and make an effective comparison with other treatment strategies.

Whatever the eventual outcome of the current clinical trials the importance of studying these very young children cannot be overstated. Population based studies show that standard treatment approaches with neurosurgery and radiotherapy are rejected, while institutional based studies repeatedly show a poor quality of outcome. These issues are concentrating the minds of paediatric oncologists, radiotherapists, and neurosurgeons to undertake rapid evaluation of the role of chemotherapy and to define an effective multidisciplinary approach to brain tumour management. To this end there is a wide range of phase I and II studies within the United Kingdom to test novel cytotoxics in childhood brain tumours. It is likely that lessons learned in these patients will affect the development of novel treatment strategies for older children.

Our thanks to members of the UKCCSG for entering patients into this study and to John Imeson, UKCCSG statistician, for calculating survival curves. The UKCCSG is supported by the Cancer Research Campaign.

1 Stiller CA, Lennox EL. Childhood medulloblastoma in Britain 1971-77: Analysis of treatment and survival. $B r \mathcal{F}$ Cancer 1983; 48: 835-41.

2 Stiller CA, Bunch KJ. Brain and spinal tumours in children under 2 years: incidence and survival in Britain, 1975-1985. Br $\mathcal{f}$ Cancer 1992; 66: suppl XVIII: 550-3. 3 Polednak AP, Flannery JT. Brain, other central nervous system and eye cancers. Cancer 1995; 75 (suppl 1): 330-7.

4 Tait DM, Thornton Jones H, Bloom HJ, et al. Adjuvant chemotherapy for medulloblastoma: the first multicentre control trial of the International Society of Paediatric Oncology (SIOP 1). Eur f Cancer 1990; 26: 464-9.

5 Belza MG, Donaldson SS, Steinberg GK, et al. Medulloblastoma in childhood: treatment results and proposal for a new staging system. Int $\mathcal{f}$ Radiat Oncol Biol Phys posal for a new stagin

6 Jenkin DJ, Goddard K, Armstrong D, et al. Posterior fossa medulloblastoma in childhood: treatment results and promedulloblastoma in childhood: treatment results and pro-

7 Silber JH, Radcliffe J, Peckham V, et al. Whole brain irradiation and decline in intelligence: the influence of dose and age on IQ score. F Clin Oncol 1993; 11: 195-6.

8 Duffner PK, Cohen ME, Thomas PRM. The long term effects of cranial irradiation on the central nervous system. Cancer 1985; 56: 1841-6.

9 Gentet JC, Doz F, Bouffet E, et al. Carboplatin and VP16 in medulloblastoma: a phase II study of the French Society of Paediatric Oncology (SFOP). Med Pediatr Oncol 1994; 23: 422-7.

10 Duffner PK, Horowitz ME, Krischer JP, et al. Postoperative chemotherapy and delayed radiation in children less than three years of age with malignant brain tumours. $N$ Engl $f$ Med 1993; 328: 1725-31.

11 Friedman HS, Krischer JP, Burger P, et al. Treatment of children with progressive or recurrent brain tumours with carboplatin and ipoplatin. $\mathcal{F}$ Clin Oncol 1992; 10: 249-56.

12 Chin HW, Maruyama Y. Recent progress and results of modern radiation therapy in medulloblastoma. Prog Clin Biol Res 1983; 130: 401-10. 
13 Hirsh JF, Renier D, Czernichow R, et al. Medulloblastoma in childhood survival and functional results. Acta Neurochir 1979; 48: 1-15.

14 Clayton PE, Shalet SM. Dose dependency of time of onset of irradiation-induced growth hormone deficiency. $\mathcal{f}$ Paediatr 1991; 118: 226-8.

15 Clayton PE, Shalet SM. The evaluation of spinal growth after irradiation. Clin Oncol 1991; 3: 220-2.

16 Duffner PK, Cohen ME, Myers MH, Heise HW. Survival of children with brain tumours. SEER programme, of children with brain tumours. SEE

17 Rosenstock JG, Evans AE, Schut L. Response to vincristine Rosenstock JG, Evans AE, Schut L. Response to vincristine 45: $135-40$

18 Allen JC, Helson L. High dose cyclophosphamide chemotherapy for recurrent CNS tumours in children. Neurosurgery 1981; 55: 749-56.

19 Walker RW, Allen JC. Cisplatin in the treatment of recurrent childhood primary brain tumours. $f$ Clin Oncol 1988; 6: 62-6.

20 Sexauer CL, Khan A, Burger PC. Cisplatin in recurrent brain tumours. A POG phase II study. Cancer 1988; 56: 1497-501.

21 Shapiro WR, Young DF, Mehta BM. Methotrexate: distribution in cerebrospinal fluid after intravenous ventricular and lumbar injection. $N$ Engl $¥$ Med 1975; 293: 161-6. 22 Schnabel A, Gross WL. Low dose methotrexate - efficacy, side effects and risk factors for side effects. Sermin Arthritis Rheum 1994; 23: 310-27.

23 Strauss LC, Killmund TM, Carsun BS, et al. Efficacy of postoperative chemotherapy using cisplatin and etoposide postoperative chemotherapy using cisplatin and etoposide in young children

24 Horowitz ME, Kun LE, Mulhern RK, et al. Feasibility and efficacy of pre-irradiation chemotherapy for paediatric brain tumours. Neurosurgery 1988; 22: 687-90.

\section{Amoebic liver abscess}

Should you happen to have Entamoeba histolytica in your bowel you would have that in common with one tenth of the people on this planet. (People on other planets rarely influence such statistics.) It is said that about $10 \%$ of those with symptomatic intestinal amoebiasis develop a liver abscess but children are affected less commonly than adults.

A report from Karachi, Pakistan, in the Paediatric Infectious Disease fournal in 1993 described 24 children with amoebic liver abscesses and now 32 children have been reported from Mexico (Giovanni Porras-Ramirez and colleagues, fournal of Pediatric Surgery 1995; 30: 662-4).

Between 1975 and 1993, 20 boys and 12 girls aged 10 months to 12 years presented to hospital in $\mathrm{La} \mathrm{Paz}$ with fever, hepatomegaly, and right upper quadrant abdominal pain and were found to have single or multiple liver abscesses. Liver ultrasound showed cystic lesions with posterior sonographic enhancement and $x$ ray films showed non-specific findings such as elevation of the right hemidiaphragm with pleural effusion. Serological testing for $E$ histolytica was positive in 30 cases.

Medical treatment was with dehydroemetine and metronidazole. In 17 children the abscess remained confined to the liver. Percutaneous needle aspiration of an abscess was performed in nine of the 17 for one of three reasons; failure to respond to medical treatment, a large $(6 \mathrm{~cm}$ or more) abscess in an ill patient, or predicted abscess rupture. An abscess was considered about to rupture when either there was erythema, swelling, and tenderness over a localised area of liver or ultrasound showed a thin walled cyst near to the liver surface. All nine of these patients did well.

Rupture of the abscess occurred in 15 cases: into the pleura in 11, the peritoneum in three, and the pericardium in one. Rupture into the pleura was treated with a chest drain, into the peritoneum with laparotomy, lavage, and drainage, and into the pericardium by pericardiotomy and later partial pericardiectomy. One child with an abscess rupturing into the pleural cavity died. All 31 survivors did well with no recurrence.

Small amoebic liver abscesses usually respond well to medical treatment. Those which don't, large ones, and those about to rupture, need aspirating. 